\title{
Genetic manipulation of RPS5 gene expression modulates the initiation of commitment of MEL cells to erythroid maturation: Implications in understanding ribosomopathies
}

\author{
IOANNIS S. VIZIRIANAKIS, ELENI T. PAPACHRISTOU, PANAGIOTIS ANDREADIS, \\ ELENA ZOPOUNIDOU, CHRISTINA N. MATRAGKOU and ASTERIOS S. TSIFTSOGLOU \\ Laboratory of Pharmacology, Department of Pharmaceutical Sciences, \\ Aristotle University of Thessaloniki, GR-54124 Thessaloniki, Greece \\ Received January 14, 2015; Accepted February 24, 2015
}

DOI: $10.3892 /$ ijo.2015.3017

\begin{abstract}
Impairment of ribosome biogenesis contributes to the molecular pathophysiology of ribosomopathies by deregulating cell-lineage specific proliferation, differentiation and apoptosis decisions of haematopoietic progenitor cells. Here, using pro-erythroblast-like murine erythroleukemia (MEL) cells, a model system of erythroid maturation, we aimed to investigate whether genetic manipulation of RPS5 expression affects the capacity of cells to grow and differentiate in culture. Parental MEL cells stably transfected with full length RPS5 cDNA in sense (MEL-C14 culture) or antisense (MEL-antisenseRPS5 culture) orientation, as well as MEL cells transiently transfected with siRNAs specific for RPS5 gene silencing (MEL-RPS5siRNA culture) were assessed for their ability to fully execute their erythroid maturation program in culture. The data obtained thus far indicate that: a) MEL-antisenseRPS5 exhibit a pronounced delay in the initiation of differentiation, as well as an impairment of commitment, since the continuous presence of the inducer in culture is required for the cells to fully execute their erythroid maturation program. b) RNAi-mediating silencing of RPS5 gene expression resulted in the inability of MEL cells to differentiate; however, when these cells were allowed to recapitulate normal RPS5 gene expression levels they regained their differentiation capacity by accumulating high proportion of erythroid mature cells. c) Interestingly the latter, is accompanied by morphological changes of cells and an impairment of their proliferation and apoptosis potential.
\end{abstract}

Correspondence to: Professor Ioannis S. Vizirianakis, Laboratory of Pharmacology, School of Pharmaceutical Sciences, Faculty of Health Sciences, Aristotle University of Thessaloniki, Thessaloniki GR-54124, Greece

E-mail: ivizir@pharm.auth.gr

Key words: murine erythroleukemia, erythroid maturation, ribosomal proteins, RPS5, antisense RNA, siRNAs, ribosomopathies
Such data for the first time correlate the RPS5 gene expression levels with the differentiation capacity of MEL cells in vitro, a fact that might also have implications in understanding ribosomopathies.

\section{Introduction}

Normal haematopoiesis during adult life in animals like in humans is a multistep complex process occurring within the bone marrow microenvironment $(1,2)$. Haematopoietic stem cells (HSCs) besides their self-renewal potential can be triggered to give rise to different blood cells (red blood cells, white blood cells, platelets) via lineage-restricted cell pathways (3-5). HSCs interact with bone marrow meschenchymal (stroma) cells as well as with growth factors and differentiation signals needed for maturation (6). During erythropoiesis, HSCs give rise to pro-erythroid progenitors which are then converted into orthochromatophilic normoblasts, subsequently reticulocytes and finally red blood cells entering the peripheral blood stream (7-10). Impairment of normal erythropoiesis is directly related to the pathophysiology of haematological disorders of metabolic or genetic nature such as porphyria, anemia, leukemia and myelodysplastic syndromes (MDS) $(11,12)$.

The induced erythroid maturation program of murine erythroleukemia (MEL) cells in culture by chemical inducers is accompanied by orchestrated gene expression patterns that involve upregulation of developmentally regulated genes and downregulation of those controlling potential for cell proliferation $(13,14)$. It has been previously reported that downregulation of genes encoding ribosomal RNAs (rRNAs) and specific ribosomal proteins (RPs), (e.g., RPS5 and RPL35a), occurs very early in MEL erythroid maturation program (15-17). At this early period (latent period; <24 h) of induction, the overall number of ribosomes also declines substantially and cells synthesize far less protein $(15,18)$. Simultaneously, a salt-labile translationally inactive form of ribosomes has been shown to exist (19). The precise molecular mechanisms underlying such progressive reduction of ribosome biogenesis and protein synthesis in mature MEL cells are still elusive; however, the assessment of ribosomal dysfunction has been considered essential in understanding the pathophysiology of 
reticulocytes disorders (20) and ribosomopathies (21). Besides, overexpression of RPS5 gene in stably transfected MEL cells (e.g., MEL-C14) has been associated with a delay in the initiation of MEL erythroid differentiation program in vitro. Interestingly, it has been demonstrated that RPS5 interferes with the ability of MEL-C14 to differentiate through perturbation of cell entrance into $G_{0} / G_{1}$ cell cycle arrest and CDK2, CDK4 and CDK6 levels (22).

In this study, we established two additional MEL cell cultures with altered RPS5 gene expression. One culture in which the cells were stably transfected with the full length of RPS5 antisense cDNA (MEL-antisenseRPS5) and another in which the cells were transiently transfected with siRNAs specific for RPS5 gene silencing (MEL-RPS5siRNA). Parental MEL and MEL cells transiently transfected with scrambled siRNAs (MEL-scrambled siRNA) were used as control cultures. Moreover, previously established MEL-C14 culture in which overexpression of RPS5 exists was also included in this study. The data obtained thus far indicate that: a) MEL-antisenseRPS5 exhibited a more pronounced delay in the initiation of differentiation program as compared to MEL-C14; b) RNAi-mediated transient silencing of RPS5 gene expression resulted in complete inability of MEL cells to differentiate in vitro; however, when these cells were permitted to restore normal RPS5 gene expression levels, their maximum differentiation capacity has been regained; c) interestingly, differentiation of MEL-antisenseRPS5 and MEL-RPS5siRNA cells was accompanied by cellular morphology changes, altered gene expression profiles and impairment of their potential for proliferation and even cell death. Overall, these findings support the concept for the first time that genetic manipulation of RPS5 gene expression level (up- and/or downregulation) critically affects the potential of MEL cells to fully complete their erythroid maturation program in vitro.

\section{Materials and methods}

Chemicals and antibodies. Dimethylsulfoxide (DMSO), hexamethylene-bis-acetamide (HMBA), benzidine dihydrocloride, vanadyl ribonucleotide complexes (VRC) and proteinase $\mathrm{K}$ was purchased from Sigma (St. Louis, MO, USA). $\left[\gamma-{ }^{32} \mathrm{P}\right]-$ dCTP (111 Tbq/mmol) was obtained from Izotop, Institute of Isotopes Co., Ltd., Budapest, Hungary, whereas the DNA ${ }^{32} \mathrm{P}$-labeling system kit was purchased from Invitrogen (Red Prime DNA Labeling System). The rabbit anti-RPS5 polyclonal antibody (pAb) raised against C-terminal oligopeptide of RPS5 was kindly provided by Dr Shuetsu Fukushi (R\&D Center, BioMedical Laboratories, Matoba, Kawagoe, Saitama, Japan). The rat anti-mouse MYC, CDK2, CDK4, CDK6 and Gata-1 mAbs were purchased from Invitrogen, Cell Signaling, Transduction Bioscience (BD) and Santa Cruz. Also, goat antimouse IgG and goat anti-rabbit IgG were obtained from Santa Cruz, respectively.

Assessment of MEL cell differentiation and the cellular content of haemoglobin. Parental MEL cells were maintained in Dulbecco's modified Eagle's medium as previously published (22). MEL cell cultures were exposed to DMSO $(1.5 \% \mathrm{v} / \mathrm{v})$ and/or HMBA $(5 \mathrm{mM})$ as indicated under individual figure. At certain time intervals upon exposure, the accumula- tion of differentiated (haemoglobin-producing; $\mathrm{Bz}^{+}$cells) cells was assessed cytochemically with benzidine $-\mathrm{H}_{2} \mathrm{O}_{2}$ solution as previously described (16). Also the haemoglobin content within the cells was determined spectrophotometrically as described elsewhere (23).

Stable transfection of MEL cells with the full length mouse RPS5 cDNA either in sense or antisense orientation. MEL-C14 cells generated by stable transfection of MEL cells with the full length mouse RPS5 cDNA to express recombinant RPS5-Myc-His protein has been previously described (22). Stable transfection of MEL cells with the full length mouse antisense RPS5 cDNA was carried out as briefly described below: logarithmically growing MEL cells were transfected with the recombinant vector pcDNA3.1(+) carrying the fulllength mouse RPS5 cDNA (715 bp in length). Such construct was generated through the ligation of the EcoRI/XhoI fragment derived from the original pBluescript $\mathrm{SK}+/$ - plasmid that carry the full length of RPS5 cDNA cloned in our laboratory (16) (GenBank accession no. Y12431) into the respective EcoRI/XhoI sites of pcDNA3.1(+) vector. The RPS5 DNA fragment was inserted into the recombinant pcDNA3.1(+) vector (Invitrogen Life Technologies, USA) in antisense orientation with respect to $\mathrm{CMV}$ promoter to generate the construct pcDNA3.1(+)-anti-RPS5. Subsequent stable transfection of MEL cells was performed by using Lipofectamine-2000 ${ }^{\mathrm{TM}}$ reagent (Invitrogen Life Technologies) and $1 \mu \mathrm{g}$ plasmid DNA of the recombinant construct pcDNA3.1(+)-anti-RPS5 according to the accompanying manufacturer's protocol. Stably transfected cells were then selected with G418, (Gibco BRL, Gaithersburg, MD, USA; $0.6 \mathrm{mg} / \mathrm{ml}$ ) added in the culture medium. G418-resistant cells outgrown from the transfected pcDNA3.1(+)-anti-RPS5 MEL culture allowed us to establish a culture designated as MEL-antisenseRPS5 and used then throughout this study. After the establishment of MEL-antisenseRPS5 culture, G418 (0.2-0.25 $\mathrm{mg} / \mathrm{ml})$ was used throughout all experiments described in this study.

Isolation of total cytoplasmic RNA, northern blot hybridization and RT-PCR analysis. Total cytoplasmic RNA isolated from control and/or inducer-treated cells at various time intervals upon incubation in culture was subjected to northern blot hybridization analysis to assess the steady-state levels of RNA transcripts encoded the RPS5 and $\beta^{\text {major }}$ globin genes, as previously described $(22,29)$.

For RT-PCR analysis, total cytoplasmic RNA (0.2-0.5 $\mu \mathrm{g})$ isolated from various MEL cell cultures was used for RT-PCR. The PCR experiments were performed by using the RobusT ${ }^{\mathrm{TM}} \mathrm{I}$ kit (Finnzymes). In detail, the primers used were: 5'-GCG GGATCCATGACTGAGTGGGAAGCA-3' (forward) and 5'-GCGGAATTCTCAGCGGTTAGACTTGGC-3' (reverse) specifically designed to allow the detection of mRNA level of endogenous RPS5 (441 bp) as previously published (22); 5'-GGACTTCGAGCAAGAGATGG-3' (forward) and 5'-AGC ACTGTGTTGGCGTACAG-3' (reverse) for $\beta$-actin (234 bp); 5'-GACCTCGACTACGACTCCGTAC-3' (forward) and 5'-CCACTGAGGGGTCAATGCAC-3' (reverse) for $c$-myc (547 bp); 5'-CTGCTGGTTGTCTACCCTTGG-3' (forward) and 5'-CCTGAAGTTCTCAGGATCCAC-3' (reverse) for $\beta^{\text {major }}$ globin (222 bp). 

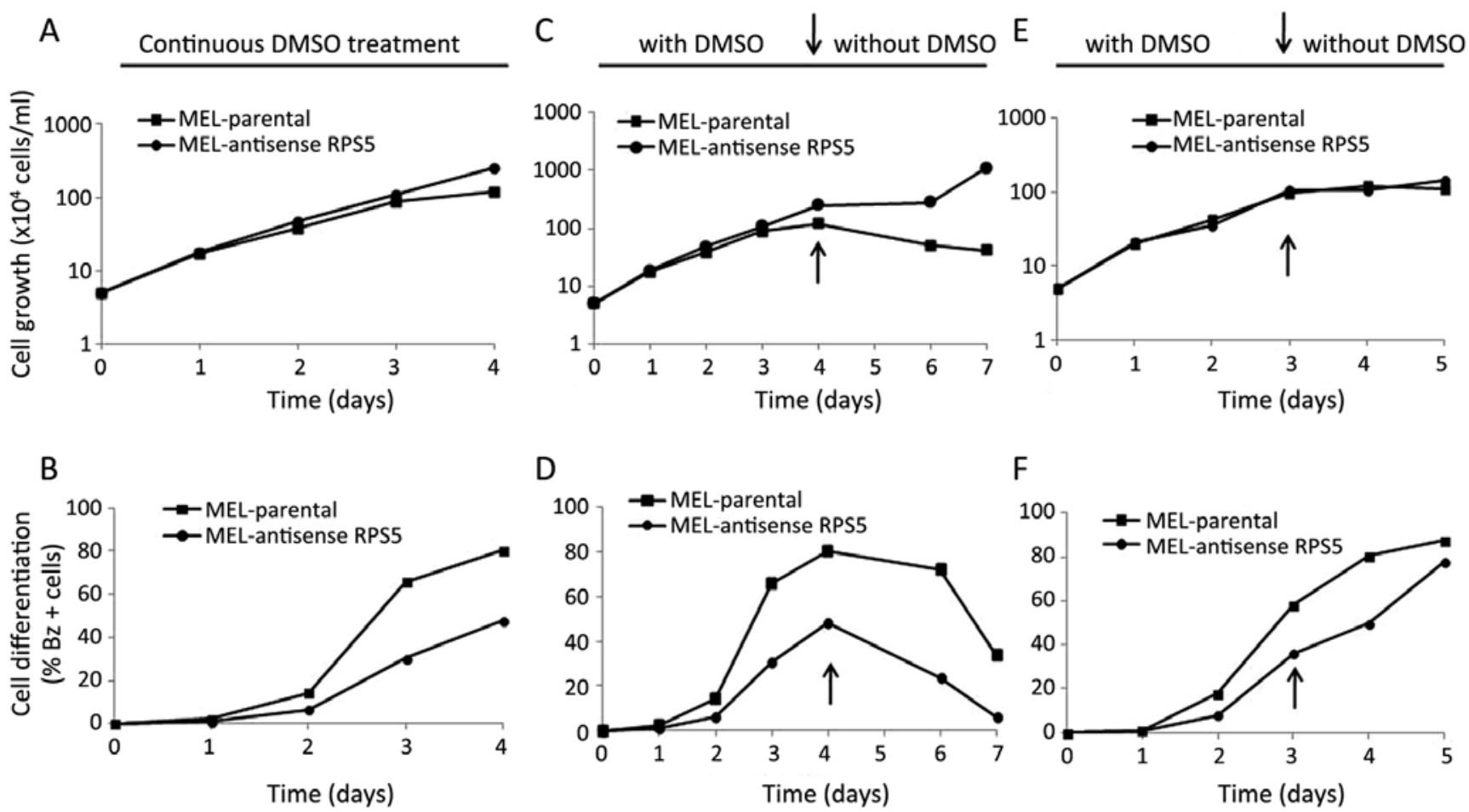

Figure 1. Assessment of proliferation and differentiation potential of MEL-antisenseRPS5 exposed to inducer DMSO upon the establishment of continuous logarithmic phase of growth with dilution with fresh medium. Parental MEL as well as MEL-antisenseRPS5 cultures (5x10 ${ }^{4}$ cells $\left./ \mathrm{ml}\right)$ were incubated either in DMEM supplemented with 10\% v/v FBS, or with DMEM supplemented with 10\% v/v FBS and G418, respectively, in the presence of the chemical inducer DMSO (1.5\% v/v). The exposure to DMSO was either continuous (A and B) without medium dilution or discontinuous with dilution with fresh medium after $4(\mathrm{C}$ and $\mathrm{D})$ or 3 days $(\mathrm{E}$ and $\mathrm{F}$ ) in order to establish logarithmic cell growth. In particular upon the 4th day, the cultures were diluted 1:10, with the addition of fresh medium in the absence of inducer DMSO, whereas as shown ( $E$ and $F$ ) and on the 3rd day, the cultures were diluted again 1:10 with the addition of fresh medium in the presence of DMSO. The kinetics of cell growth (A, C and E) and differentiation (B, D and F) was assessed as indicated in Materials and methods. The data shown are from a representative experiment repeated at least three times and the numbers represent the mean of two independent measurements.

Transfection with scrambled and RPS5 specific siRNAs. All siRNAs used including a negative control siRNA [negative control siRNA (\#1022076)] designed with no significant homology to any sequence in the mouse genome (scrambled siRNA) along with two predesigned siRNAs directed against mouse RPS5 (NM_009095) (Mm_Rps5_4 \#SI01407322; Mm_Rps5_4 \#SI01407336) were obtained from Qiagen. Based on extensive preliminary data (not shown), however, only Mm_Rps5_4 was capable of specifically downregulating the expression of RPS5 gene in parental MEL cells and, therefore, was used throughout this study. Scrambled and Mm_Rps5_4 siRNAs were transfected in MEL cells using HiPerFect reagent (Qiagen). The oligo-nucleotide sequence for siRNAs included in Mm_Rps5_4 were: sense, r(GCGCUU CCGCAAAGCACAA)dTdT; antisense, r(UUGUGCUUU GCGGAAGCGC)dTdT), whereas in scrambled siRNA were sense, UUCUCCGAACGUGUCACGUdTdT; antisense, ACG UGACACGUUCGGAGAAdTdT. The procedure briefly was as follows. The day before transfection, MEL cells were seeded at a density of $0.4-1.6 \times 10^{6}$ cells in $60-\mathrm{mm}$ dishes with $4 \mathrm{ml}$ of DMEM containing serum and antibiotics. On the day of transfection either RPS5 specific or scrambled siRNA were diluted in culture to give a final concentration of $120 \mathrm{nM}$. The transfection of siRNAs was facilitated by adding $20 \mu \mathrm{l}$ of HiPerFect reagent buffer according to the manufacturer's protocol and the cells were further incubated under normal growth conditions before silencing of the RPS5 gene to be monitored by RT-PCR analysis.

Flow cytometry DNA analysis and assessment of apoptosis. Samples of parental MEL cells as well from those exposed either to specific RPS5 (MEL-RPS5siRNA) or scrambled (MEL-scrambled-siRNA) siRNAs before harvested from culture at time intervals as shown in each figure, were subjected to flow cytometry DNA analysis and assessment of apoptosis, as previously published $(22,24)$.

\section{Results}

Stable transfection of mouse antisense RPS5 cDNA affects the commitment and decreases the onset of MEL cells to erythroid maturation in vitro. Stable transfection of MEL cells with the mouse antisense RPS5 cDNA allowed us to assess the proliferation and differentiation behavior of such cells in culture. As shown in Fig. 1B, MEL-antisenseRPS5 cells failed to reach the maximum differentiation level upon exposure to chemical inducer DMSO as compared to parental MEL cells, although comparatively no significant change on their cell growth capacity was observed (Fig. 1A). In particular, parental MEL cells exhibited high levels of $\mathrm{Bz}^{+}$(haemoglobin-producing) cells after 96-h exposure to DMSO (>80\%) as expected (22). On the contrary, MEL-antisenseRPS5 culture has shown a delay 
of 18-24 $\mathrm{h}$ in the initiation of differentiation and the proportion of $\mathrm{Bz}^{+}$cells was $\sim 50 \%$ (Fig. 1B). These data suggest that stable transfection of MEL cells with antisense RPS5 cDNA delayed the onset of differentiation by reducing the number of terminally differentiated cells accumulated in culture.

By being aware on how the commitment to erythroid maturation proceeds in MEL cells, a more thorough kinetic analysis was performed in cultures to better understand the behavior of MEL-antisenseRPS5. In particular it has been reported earlier that in parallel to haemoglobin synthesis, commitment of inducer-treated cells to terminal erythroid maturation leads to limitation of proliferation potential of MEL cells up to 4-5 cellular divisions $(13,14)$. This event becomes quite clear upon dilution of cultures of inducer-treated cells. Such dilution approach has been successfully applied in our work in MEL-C14 culture overexpressing the recombinant RPS5-Myc-His protein (22). Inducer-treated cells, fail to grow and divide continuously in contrast to uncommitted-undifferentiated MEL cells, which exhibit an unlimited proliferation potential. To this end, MEL-antisenseRPS5 cells were exposed continuously to inducer DMSO for either 96 or $72 \mathrm{~h}$, before the dilution of cultures with fresh medium to permit cells to proliferate for further 3 days in the absence (Fig. 1C and D) or 2 days in the presence of DMSO (Fig. 1E and F), respectively. Under these conditions, cells are capable of exhibiting their maximum proliferation potential by executing the erythroid maturation program in the absence of the inducer. As shown in Fig. 1C, parental MEL cells initially growing for $96 \mathrm{~h}$ in the presence of DMSO continued in the absence of DMSO for additional $72 \mathrm{~h}$, they reached their plateau phase of growth and maintained at that level despite the dilution (1:10) of the culture with fresh medium. The latter result is attributed to the differentiation-dependent restriction of proliferation potential of DMSO-treated cells $(13,14,22)$, since they achieved high level of differentiated cells $(>80 \%)$ after $96 \mathrm{~h}$ in culture (Fig. 1D). However, the proportion of differentiated cells decreased thereafter upon dilution as expected, since the committed cells can go on only for 4-5 divisions, as mentioned above. On the contrary, MEL-antisenseRPS5 cells did not exceed the level of $50 \%$ of differentiated cells under the same culture conditions (Fig. 1D). Moreover in this culture, cellular growth increased after the 6th day (Fig. 1C), since the higher number of non-committed cells $(\sim 50 \%)$ continued to grow in the absence of DMSO, as expected. These data suggest that in MEL-antisenseRPS5 culture fewer cells enter the differentiation program at a slower rate. Such observation proposes that the plateau phase of growth seen in antisense-RPS5-transfected MEL cells before dilution has to be attributed to cell-cell contact inhibition rather than differentiation-dependent restriction of cell proliferation potential, as seen in parental MEL cells. To confirm this observation, the same experiment was repeated but allowing cells to grow in the presence of DMSO even after dilution of cultures. Interestingly enough, in that case the MEL-antisenseRPS5 cells reached the same high level of differentiation ( $>80 \%$ ) after 5 days in culture compared to parental MEL, as illustrated in Fig. 1E and F. These results suggest that the onset of differentiation has been significantly delayed by the transfection of antisense-RPS5 cDNA and the presence of inducer is required throughout the entire exposure period for MEL cells to complete their erythroid maturation program. At this point it has to be noted that such behavior is unique for MEL-antisenseRPS5 culture, since both parental MEL and MEL-C14 did not require the presence of inducer after initiation of commitment to execute the differentiation program, as previously published (22).

Correlation of RPS5 gene expression with modulation of CDKs in DMSO-treated MEL-antisenseRPS5. To assess any changes in the steady-state level of RPS5 RNA transcripts in MEL-antisenseRPS5 cells in proliferating and/ or differentiating cultures, isolated cytoplasmic RNA was subjected to northern blot hybridization analysis using $\beta^{\text {major }}$ globin gene as internal control. As shown in Fig. 2A, the level of RPS5 RNA transcripts in the cytoplasm remained constant in MEL-antisenseRPS5 cells growing in culture in the absence of DMSO. Similar data were obtained even after 96-h exposure to DMSO (Fig. 2C). On the contrary, the gradual cytoplasmic accumulation of $\beta^{\text {major }}$ globin RNA transcripts was evident in differentiating cells only after 48-72 h (Fig. 2D). The latter, represents a difference in what was seen in differentiating parental MEL cells where a substantial decrease of RPS5 mRNA after $24 \mathrm{~h}$ and a significant accumulation of $\beta^{\text {major }}$ mRNA after $36 \mathrm{~h}$ has been detected $(16,22)$. These marginal differences seen in the expression level of both RPS5 and $\beta^{\text {major }}$ globin genes in differentiating MEL-antisenseRPS5 cells seems more likely to be related to their limited commitment potential to erythroid maturation, as shown in Fig. 1.

By knowing that commitment of MEL cells to erythroid maturation is correlated with cell cycle changes, the assessment of the expression profile of endogenous RPS5 and CDKs (CDK2, CDK4 and CDK6) upon differentiation of MEL-antisenseRPS5 cells was investigated. During the course of differentiation, the Gata-1 protein level was also assessed as an internal control for MEL cells, as previously described (22). Cell lysates prepared from differentiating MEL-antisenseRPS5 cultures and subjected to immunoblotting revealed that the RPS5 protein level decreased quite early after $24 \mathrm{~h}$ (Fig. 2F). This is interesting, since in parental MEL cells the decrease of RPS5 protein is detected only after $72 \mathrm{~h}$ (22). Regarding the assessment of CDKs, the level of CDK2 and CDK4 proteins remained almost constant, whereas that of CDK6 decreased very early $\sim 2 \mathrm{~h}$ after the induction of differentiation (Fig. 2G-I). These differences seen in the expression level of CDKs seems to be correlated with the differentiation potential of MEL-antisenseRPS5 cells, since the expression profile of Gata-1 remained almost constant throughout the course of the experiment (Fig. 2J). The expression profile of CDKs detected at late stages of differentiating MEL cells coincide with previous observations (22,25-28).

In an effort to rule out the possibility that the delay observed in the onset of differentiation of MEL-antisenseRPS5 cells could be attributed to inducer DMSO, the chemical inducer hexamethylene-bis-acetamide (HMBA) has been also included for further analysis $(13,14)$. Based on the experimental data obtained, a decrease in the onset of differentiation was also recorded since the number of differentiating MEL-antisenseRPS5 cells accumulated in culture after $96 \mathrm{~h}$ is less $(\sim 40 \%)$ as compared to that seen in parental MEL cells (>80\%) (Fig. 3E). Interestingly, this effect was more pronounced 
Control-untreated
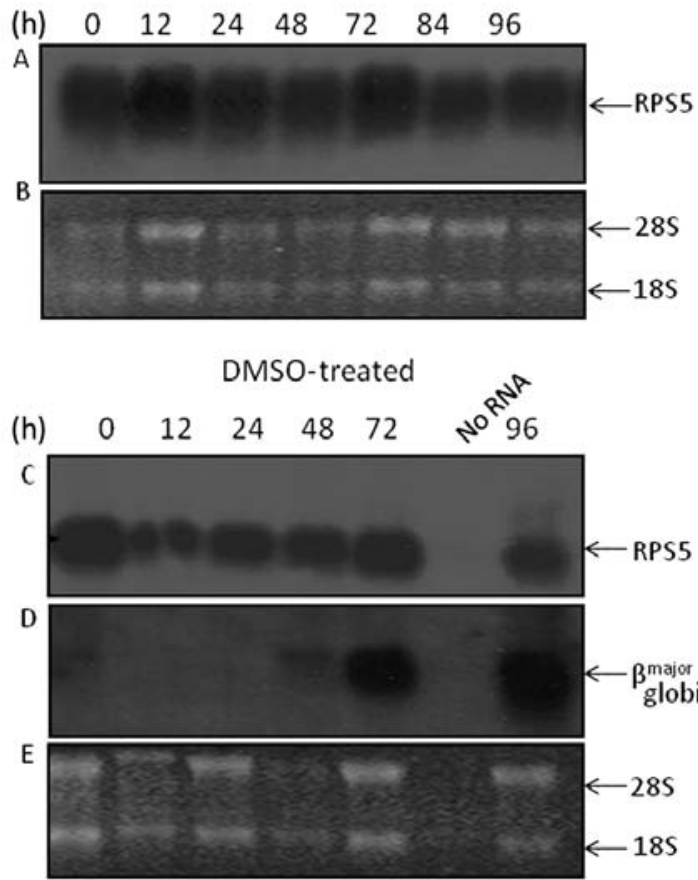

DMSO-treated
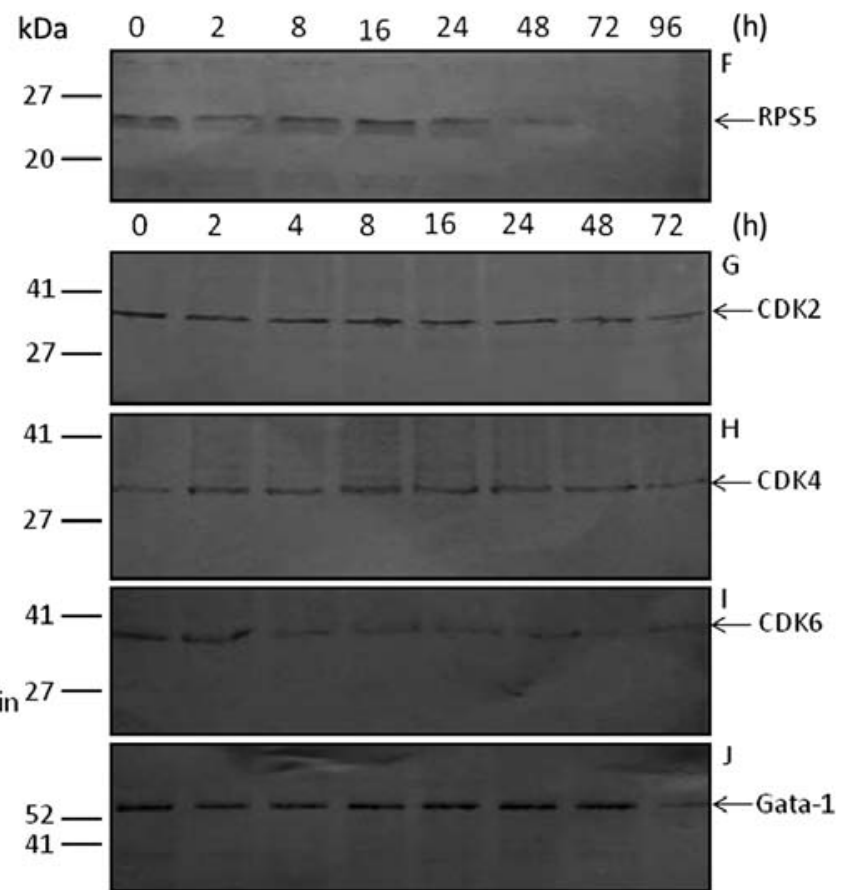

Figure 2. Assessment by northern blot hybridization of the steady-state level of RPS5 and $\beta^{\text {major }}$ globin RNA transcripts as well as RPS5 and CDKs protein level in MEL-antisenseRPS5 cell cultures grown in the presence of DMSO. MEL-antisenseRPS5 cell cultures were grown in DMEM supplemented with $10 \% \mathrm{v} / \mathrm{v}$ FBS and G418 in the absence (control-untreated) or presence (DMSO-treated) of DMSO (1.5\% v/v). At times indicated, cells were harvested from culture and total cytoplasmic RNA and cellular protein extracts were isolated, as described in Materials and methods. (A, C and D) Cytoplasmic RNA $(10 \mu \mathrm{g})$ was electrophoretically separated on $1 \%$ agarose gel, transferred onto a nylon membrane, and hybridized at $65^{\circ} \mathrm{C}$ with $\left.{ }^{32} \mathrm{P}\right]-\mathrm{labeled} \mathrm{DNA}$ fragments coding for mouse RPS5 mRNA (715 bp) (A and C) and/or $\beta^{\text {major }}$ globin gene (7,304-bp genomic DNA fragment containing the entire gene) (D). The ethidium-bromide staining pattern of the isolated cytoplasmic RNA transcripts from control-untreated (B) and DMSO-treated (E) MEL-antisenseRPS5 cells is shown. The position of the $28 \mathrm{~S}$ and $18 \mathrm{~S}$ rRNAs is indicated by the arrows. (F-J) Western blot analysis using $30 \mu \mathrm{g}$ protein extracts from each culture preparation was carried out for the assessment of RPS5 (F), CDK-2 (G), CDK-4 (H), CDK-6 (I) and Gata-1 (J) protein levels. Abs for RPS5 (C-terminal), CDK2, CDK-4 and CDK-6 (1:1,000 dilution) and for Gata-1 (1:400 dilution) was used for immunoblotting, as shown in Materials and methods.

\section{Control untreated MEL cells}

A

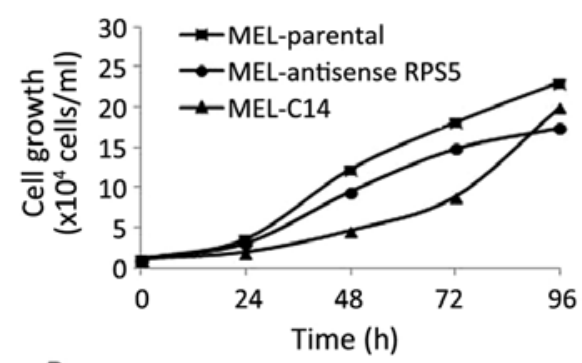

B

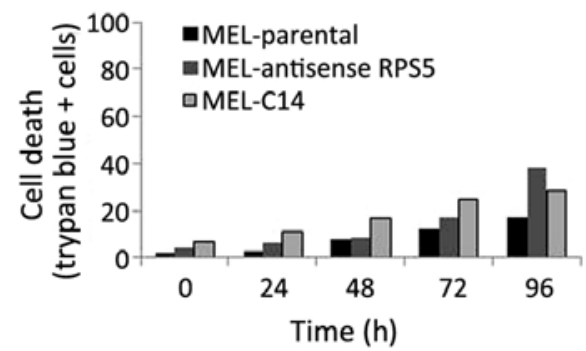

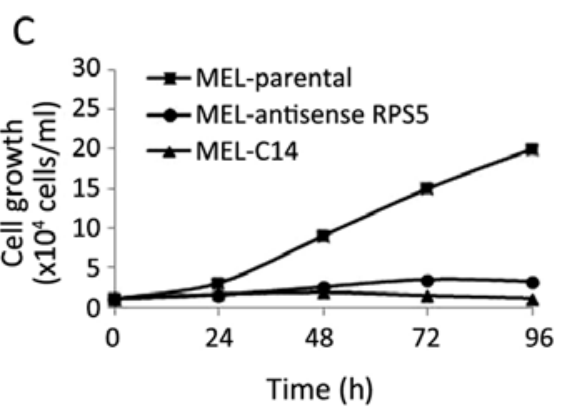

D

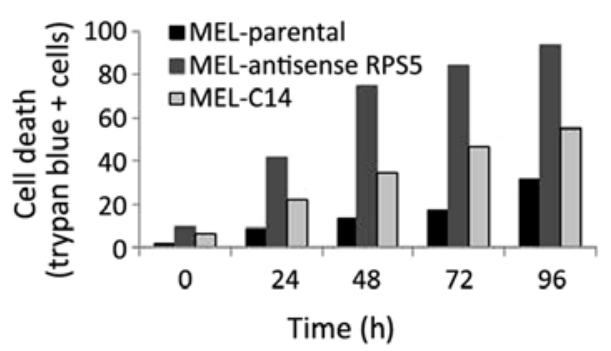

HMBA treated MEL cells

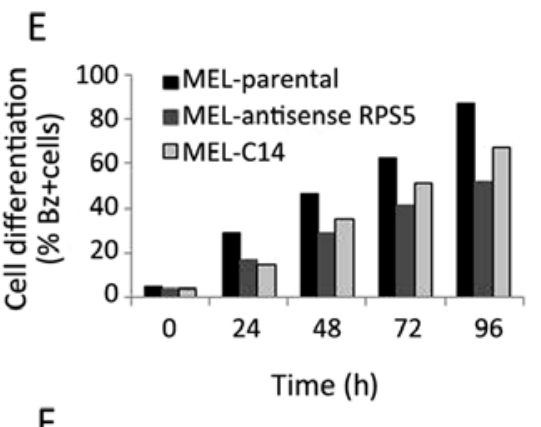

$\mathrm{F}$

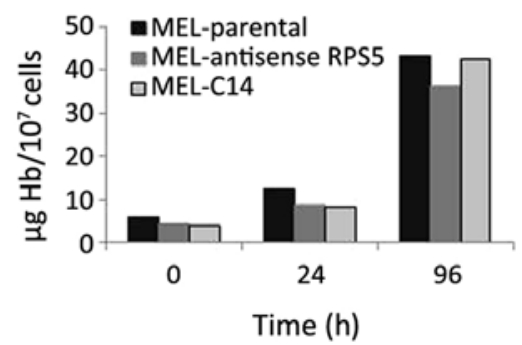

Figure 3. Assessment of proliferation and differentiation potential of MEL-antisenseRPS5 exposed to inducer HMBA. Parental MEL and MEL-antisenseRPS5 cultures $\left(5 \times 10^{4}\right.$ cells $\left./ \mathrm{ml}\right)$ were incubated either in DMEM supplemented with $10 \% \mathrm{v} / \mathrm{v}$ FBS, or with DMEM supplemented with $10 \%$ v/v FBS and G418 $(0.25 \mathrm{mg} / \mathrm{ml})$, respectively, in the absence (A and B) or the presence (C-F) of the chemical inducer HMBA $(5 \mathrm{mM})$. The kinetics of cell growth (A and C), cell death (B and D), differentiation (E) and haemoglobin content $(F)$ was assessed as indicated in Materials and methods. The data shown are from a representative experiment repeated at least three times and the data represent the mean of two independent measurements. 


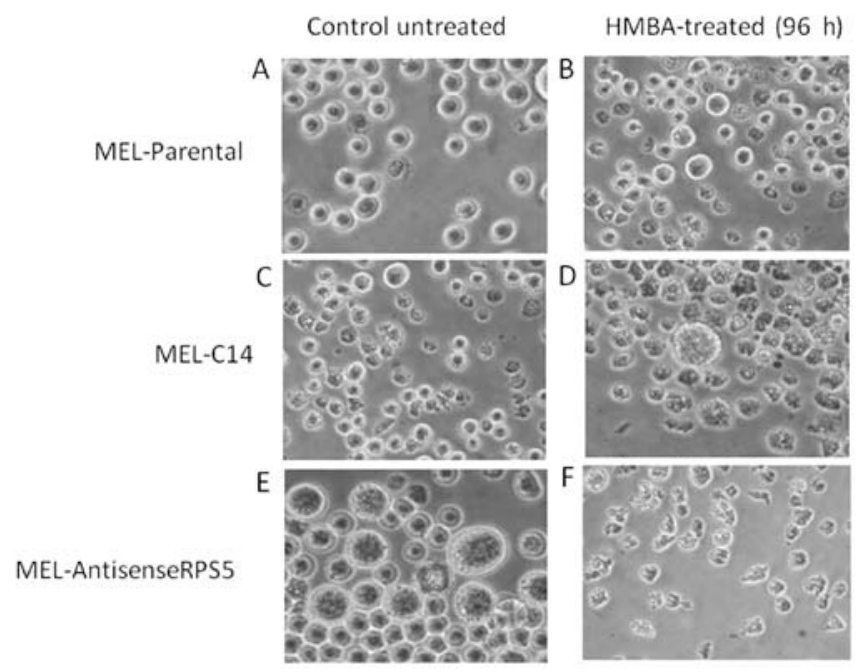

Figure 4. Cellular morphology of control-untreated or HMBA-treated cultures of MEL parental, MEL-C14 and/or MEL-antisenseRPS5. MEL parental, MEL-C14 and MEL-antisenseRPS5 cultures were grown in the absence (control-untreated) or the presence of inducer HMBA $(5 \mathrm{mM})$ for $96 \mathrm{~h}$ (HMBA-treated). The morphology of cells was assessed by light microscopy. Cell images were taken with a Nikon Coolpix P5100 camera adjusted on a Nikon Eclipse TS100 light microscope. The images obtained are presented $\mathrm{x} 60$.

even by comparing the number of differentiating cells seen in MEL-C14 cells ( $60 \%)$. The latter culture, as reported earlier, also exhibits a delay in the initiation and the onset of differentiation upon treatment with HMBA attributed to the overexpression of recombinant RPS5-Myc-His protein (22). Moreover, the amount of haemoglobin accumulated in differentiating MEL-antisenseRPS5 is lower than that measured in parental MEL and MEL-C14 cultures (Fig. 3F). In addition, cell growth is decreased dramatically in MEL-antisenseRPS5 culture upon exposure to HMBA similarly to MEL-C14 (Fig. 3C), whereas the number of non-viable cells is much higher in MEL-antisenseRPS5 ( 85\%) compared to both parental MEL ( $\sim 25 \%)$ and MEL-C14 ( 45\%) cells (Fig. 3D). However, these differences in cell growth capacity and viability recorded in cultures is more likely to be attributed to their differentiation potential, since the untreated-control cells behave similarly and achieve comparable high numbers of proliferating and viable cells after $96 \mathrm{~h}$ (Fig. 3A and B). This notion is further supported by examining the morphology of cells in culture as well as assessing the gene expression profile of $\beta$-actin (housekeeping gene), RPS5 (gene of interest) and $\beta^{\text {major }}$ globin (a developmentally regulated gene) upon induction of differentiation of MEL-antisenseRPS5. Indeed, the morphology of untreated MEL-antisenseRPS5 exhibit an altered phenotype with a larger size that, however, upon 96-h exposure to inducing agent HMBA is further exacerbated by giving smaller although abnormally differentiated cells (distorted nucleolus and nucleus) (Fig. 4). Moreover, a dramatic decrease in mRNA levels for all three genes was observed after 48-72-h exposure of cells to HMBA (data not shown), whereas no significant alteration was seen for RNA transcripts of $\beta$-actin, $\beta^{\text {major }}$ globin and c-myc (an oncogene with crucial role in the differentiation potential of MEL cells) in control-untreated cells continuously growing in culture even for $120 \mathrm{~h}$. Such data collectively propose a correlation between antisense-RPS5 transfection and the decrease in the onset of differentiation seen in MEL-antisenseRPS5 cell culture, since dismantling of the differentiation program is observed very early regardless of the nature of the chemical inducer (DMSO and/or HMBA) employed.

RNAi-mediated silencing of RPS5 gene expression blocked $M E L$ cell differentiation in vitro. In order to more thoroughly investigate the correlation of RPS5 gene expression with the capacity of MEL cells to initiate their erythroid maturation program in vitro, we applied the RNA interference (RNAi) methodology to specifically downregulate the expression of RPS5 in this culture. As shown in Fig. 5, by using specific siRNAs the silencing of RPS5 gene in MEL cells was achieved in both mRNA and protein level after 24 and $36 \mathrm{~h}$, respectively (Fig. 5A and C). Interestingly, this effect was evident even after 72-96-h continuous exposure to specific siRNAs for the RPS5 gene and not for the scrambled siRNAs used as control (data not shown). The successful RNAimediated silencing of RPS5 gene in MEL cells prompted us to assess the capacity of such culture to differentiate in vitro upon exposure to the chemical inducer HMBA. The experimental design shown in Fig. 6 allowed us to verify for each experiment the successful RPS5 gene silencing and also to assess the differentiation capacity of such a culture (Fig. 6A). In
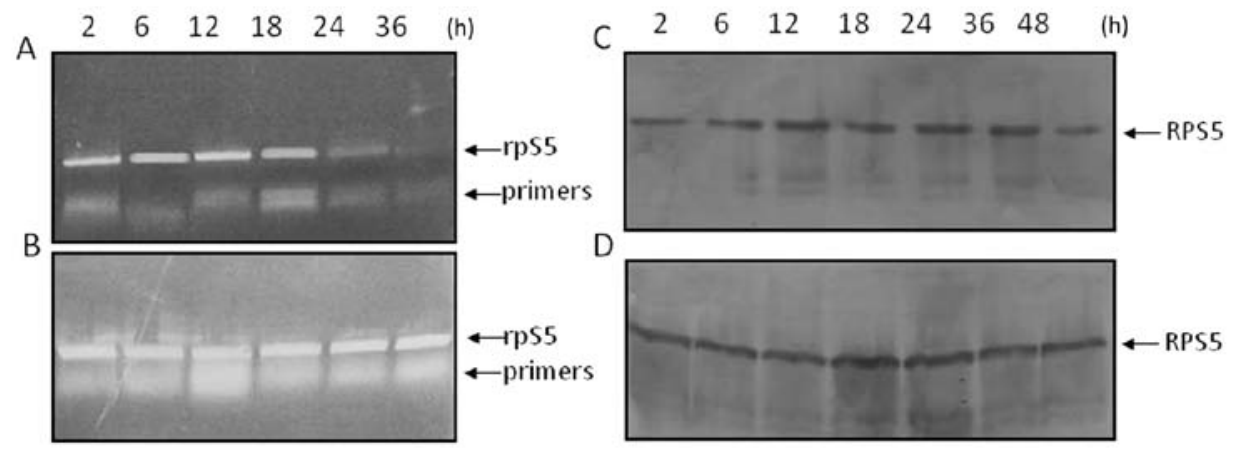

Figure 5. Time course assessment of RNAi-mediated RPS5 gene silencing in parental MEL cells. Parental MEL cells grown in culture were exposed with either scrambled (B and D) or RPS5 specific (A and C) siRNAs as indicated in Materials and methods. At time intervals indicated, cells were harvested from culture and total cytoplasmic RNA and protein extracts were isolated and subjected to either RT-PCR [(A and B) $0.5 \mu \mathrm{g}$ RNA] or western blot [(C and D) $20 \mu \mathrm{g}$ protein] analysis for RPS5 gene and protein expression, respectively, as described in Materials and methods. 

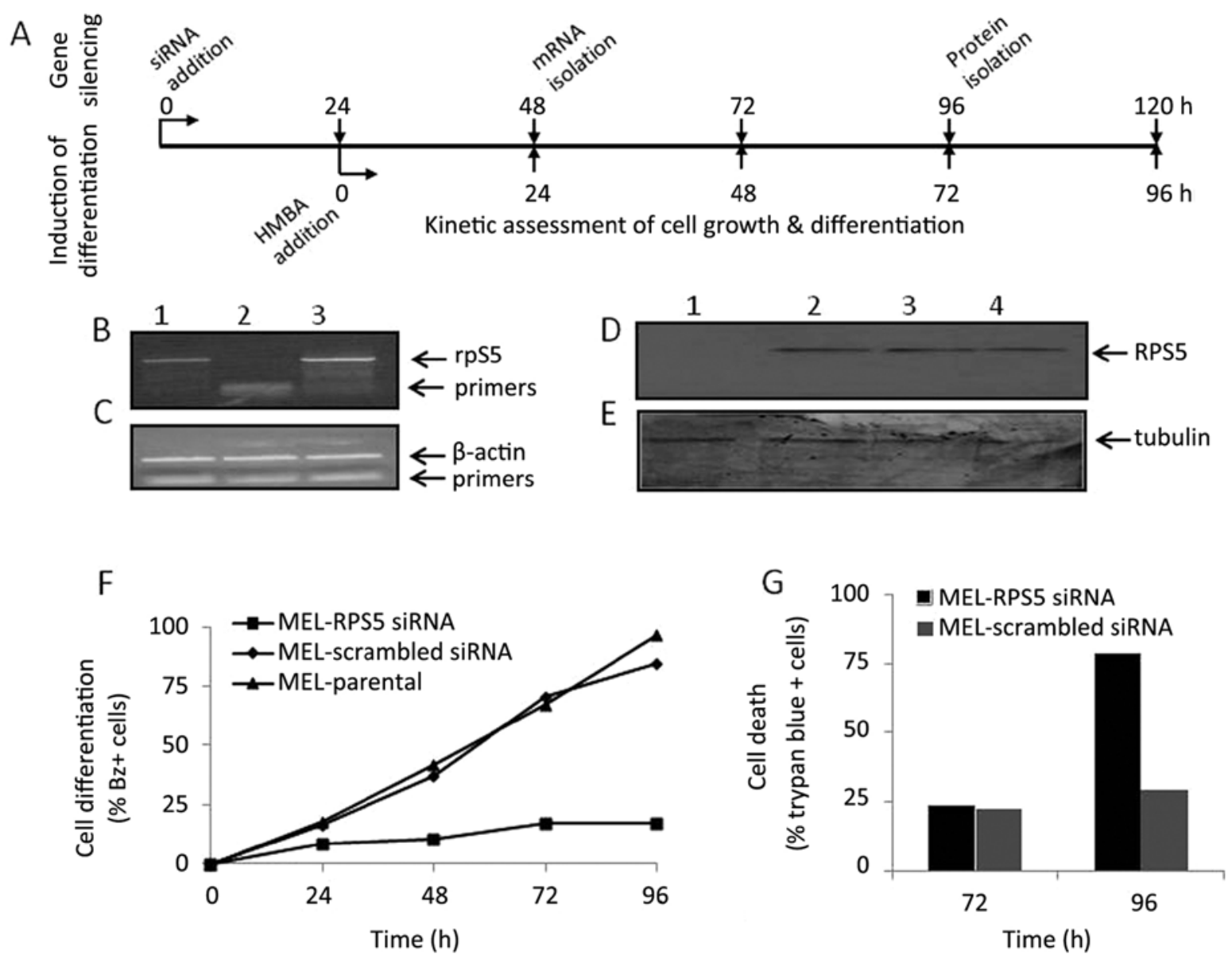

Figure 6. Assessment of differentiation potential of parental MEL cells treated with RPS5 specific siRNAs. Parental MEL cells were treated with either scrambled (MEL-scrambled siRNA) or RPS5 specific (MEL-RPS5 siRNA) siRNAs as indicated in Fig. 4 for 24 h, before the addition of inducer HMBA $(5 \mathrm{mM})$. Note that parental MEL cells treated without siRNA molecules (MEL parental) exposed to HMBA were also included in this experimental design as control. Afterwards, the number of cells producing haemoglobin (benzidine-positive cells; differentiated cells) accumulated in culture was assessed kinetically for a 96-h period (F), as described in Materials and methods. After 48- and 96-h continuous exposure to siRNAs in culture, as shown diagrammatically (A), isolation of total cytoplasmic RNA and protein extracts was carried out to assess the expression RPS5 gene and protein level by RT-PCR [(B) $0.5 \mu \mathrm{g}$ RNA] and western blotting [(D) $20 \mu \mathrm{g}$ protein], respectively. The assessment of $\beta$-actin mRNA (C) and tubulin protein (E) was also carried out and used as controls. (G) The accumulation of trypan-blue (dead) staining cells was measured after 72- and 96-h exposure of cells to the inducer HMBA (A). (B and C) Cultures treated with HMBA for $24 \mathrm{~h}$; lane 1, MEL parental; lane 2, MEL-PRS5 siRNA; lane 3, MEL-scrambled siRNA. (D and E) Cultures treated with (lanes 1-3) or without (lane 4) the presence of HMBA for $72 \mathrm{~h}$; lane 1, MEL-RPS5 siRNA; lane 2, MEL-scrambled siRNA; lanes 3 and 4, parental MEL. The data shown are from a representative experiment repeated at least three times and the data represent the mean of two independent measurements.

particular, the cells were initially exposed to suitable siRNAs (scrambled and/or RPS5-specific) for $24 \mathrm{~h}$ before the addition of HMBA in culture. After an additional period of $24 \mathrm{~h}$, the silencing of RPS5 gene expression was verified by RT-PCR, as shown in Fig. 6B. The RPS5 protein level was not detectable after 96-h exposure to RPS5-specific siRNAs, in contrast to cultures exposed to scrambled siRNAs (Fig. 6D). Importantly however, the monitoring of differentiation potential of these cultures for the entire 96-h exposure period to inducer HMBA indicated that only MEL cells exposed to RPS5-specific siRNAs failed to initiate their erythroid maturation program in vitro (Fig. $6 \mathrm{~F}$ ). The number of differentiating cells (cells producing haemoglobin) accumulated after $96 \mathrm{~h}$ in culture exposed to specific RPS5 siRNAs is very low ( $15 \%)$, whereas MEL cells exposed to scrambled siRNAs reach a level comparable to parental ones (>85\%). In parallel, assessment of trypan-blue positive cells in these cultures revealed that in both conditions the number of dead cells marginally exceed $20 \%$ after 72 -h exposure to HMBA; however, after $96 \mathrm{~h}$ in culture only MEL cells exposed to RPS5-specific siRNAs exhibited a high proportion (80\%) of dead cells (Fig. 6G).

MEL cultures exposed to RPS5-specific siRNAs and being induced to differentiate by HMBA exhibit different cellular morphology. In particular, parental and scrambled siRNAtreated MEL cells showed the typical erythroid maturation phenotype as expected after 72-96 h. However, differentiating cells exposed to RPS5-specific siRNAs present a phenotype more resembling that of undifferentiated cells where nucleolus and nucleus are distorted (data not shown). The latter, is in accordance with the inability of such a culture to initiate commitment of erythroid maturation and accumulate high proportion of haemoglobin-producing cells, as presented above. In order to clarify such differentiation behavior of cells, the assessment of the expression level of RPS5, c-myc, $\beta^{\text {major }}$ globin and $\beta$-actin genes was further investigated. Upon induction of MEL cell differentiation with HMBA, parental MEL, MEL-C14 and MEL-scrambled siRNA-treated cells exhibited decreased level of RPS5 (data not shown). On the 
HMBA-

treatment

$24 \mathrm{~h}$

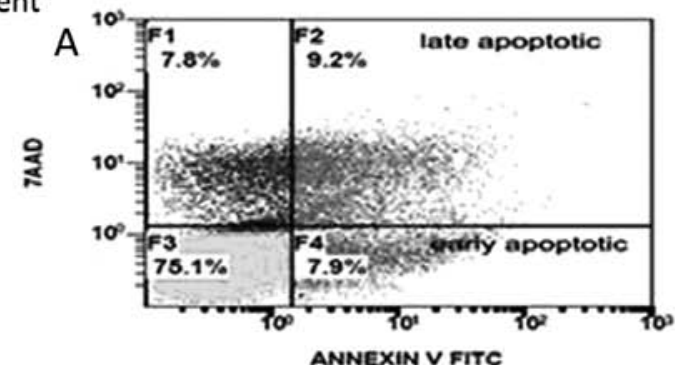

$48 \mathrm{~h}$

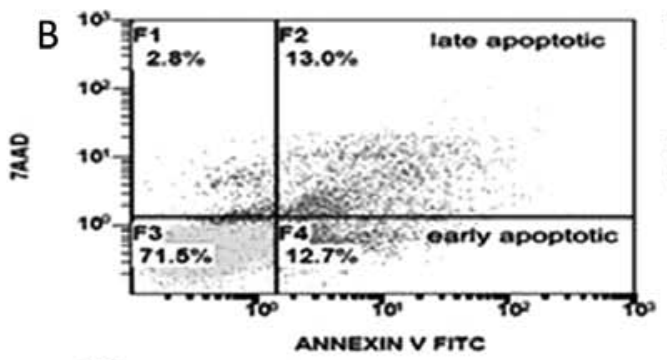

$72 \mathrm{~h}$

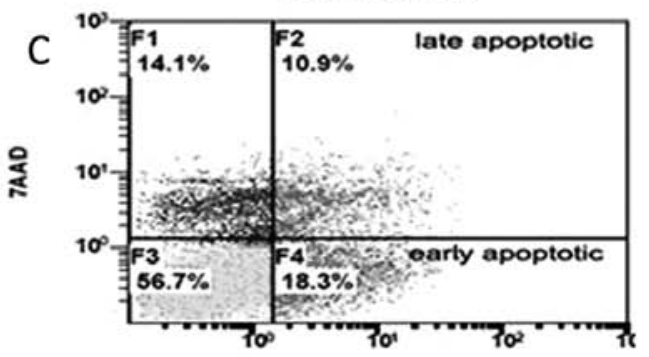

ANNEXIN $\checkmark$ FITC

$96 \mathrm{~h}$

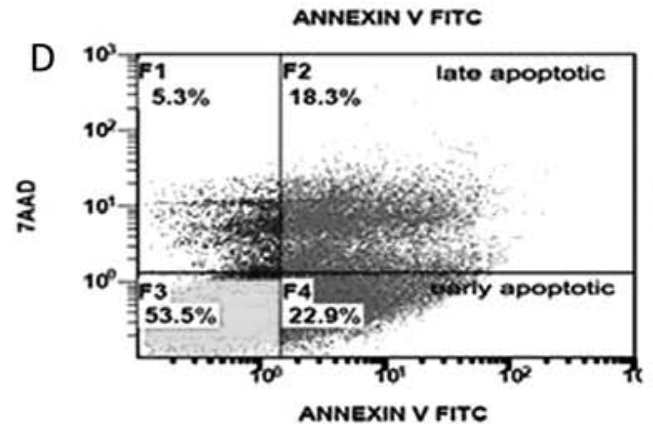

MEL-RPS5 SiRNA

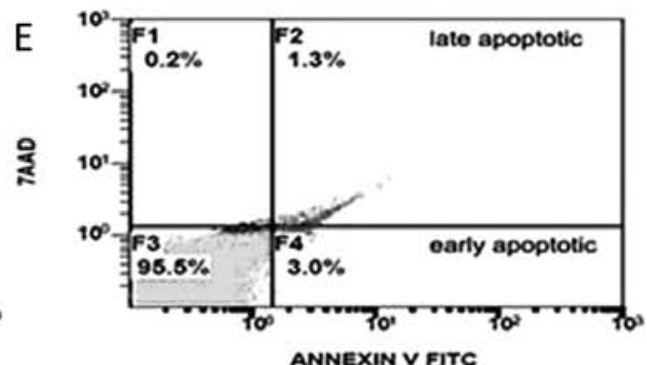

$\mathrm{F}$
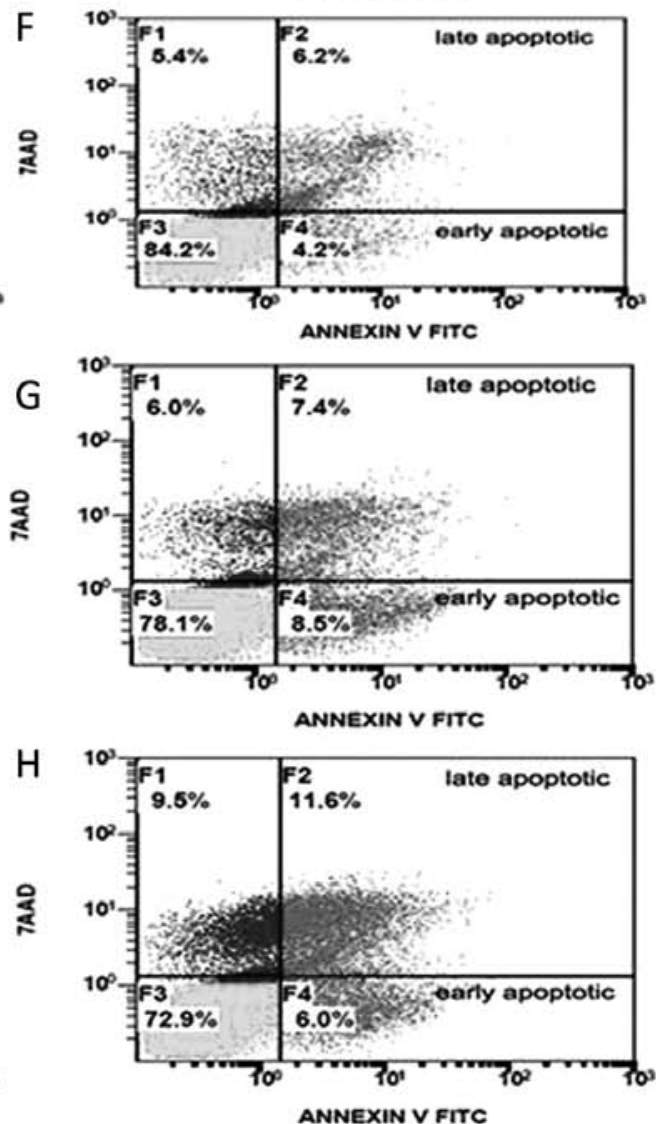

Figure 7. Flow cytometry analysis for the assessment of apoptotic cells in MEL-scrambled siRNA and MEL-RPS5 siRNA cultures exposed to HMBA. MEL-scrambled siRNA and MEL-RPS5 siRNA cells grown in the presence of HMBA (5 mM) as shown in Fig. 6 harvested from culture at time intervals indicated, washed, stained with Annexin V/AAD reagent and then processed for flow cytometry, as described in Materials and methods. The data obtained for apoptotic and non-viable cells is indicated.

contrary, the steady-state level of RPS5 RNA transcripts in MEL-siRNA RPS5 culture was much lower from the beginning and throughout the entire exposure period, as expected. The steady-state mRNA level of $c$-myc in parental MEL showed an early initial increase at $6 \mathrm{~h}$ before a final decline after $48 \mathrm{~h}$, in agreement with a previous report (29). In MEL-C14 culture, the steady-state $c$-myc RNA transcripts indicated a biphasic profile, where an initial downregulation was observed very early $(3 \mathrm{~h})$ followed by an increase after $24 \mathrm{~h}$ before the final decrease afterwards (data not shown). Moreover, in both MEL-scrambled siRNA and MEL-siRNA RPS5 cultures, the low expression level of c-myc at 24-48 $\mathrm{h}$ was followed by a significant increase at $72 \mathrm{~h}$ before a final decrease later on. In parallel, the level of $\beta^{\text {major }}$ globin gene expression in differentiating parental MEL, MEL-C14 and
MEL-scrambled siRNAs cells showed a time-dependent increase, as expected, with the higher level seen after $96 \mathrm{~h}$ in all cultures. Importantly, however, the level of $\beta^{\text {major }}$ globin gene expression in differentiating MEL-RPS5 siRNA exhibited no substantial change by being kept at low levels even after $96 \mathrm{~h}$ as compared to all other cultures (data not shown). Furthermore, the level of $\beta$-actin mRNA did not show any significant change either between the four cultures or during the course of differentiation.

Restoration of normal RPS5 expression levels in RNAimediated RPS5 gene silencing MEL cells leads the culture to regain maximum erythroid maturation capacity in vitro. The commitment of MEL cells to erythroid maturation is associated with early accumulation of cells in $\mathrm{G}_{0} / \mathrm{G}_{1}$ cell cycle 
A

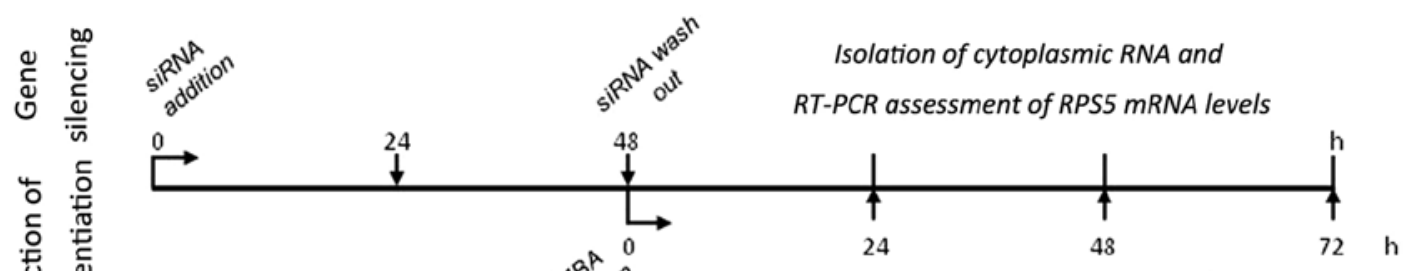

Kinetic assessment of cell growth \& differentiation
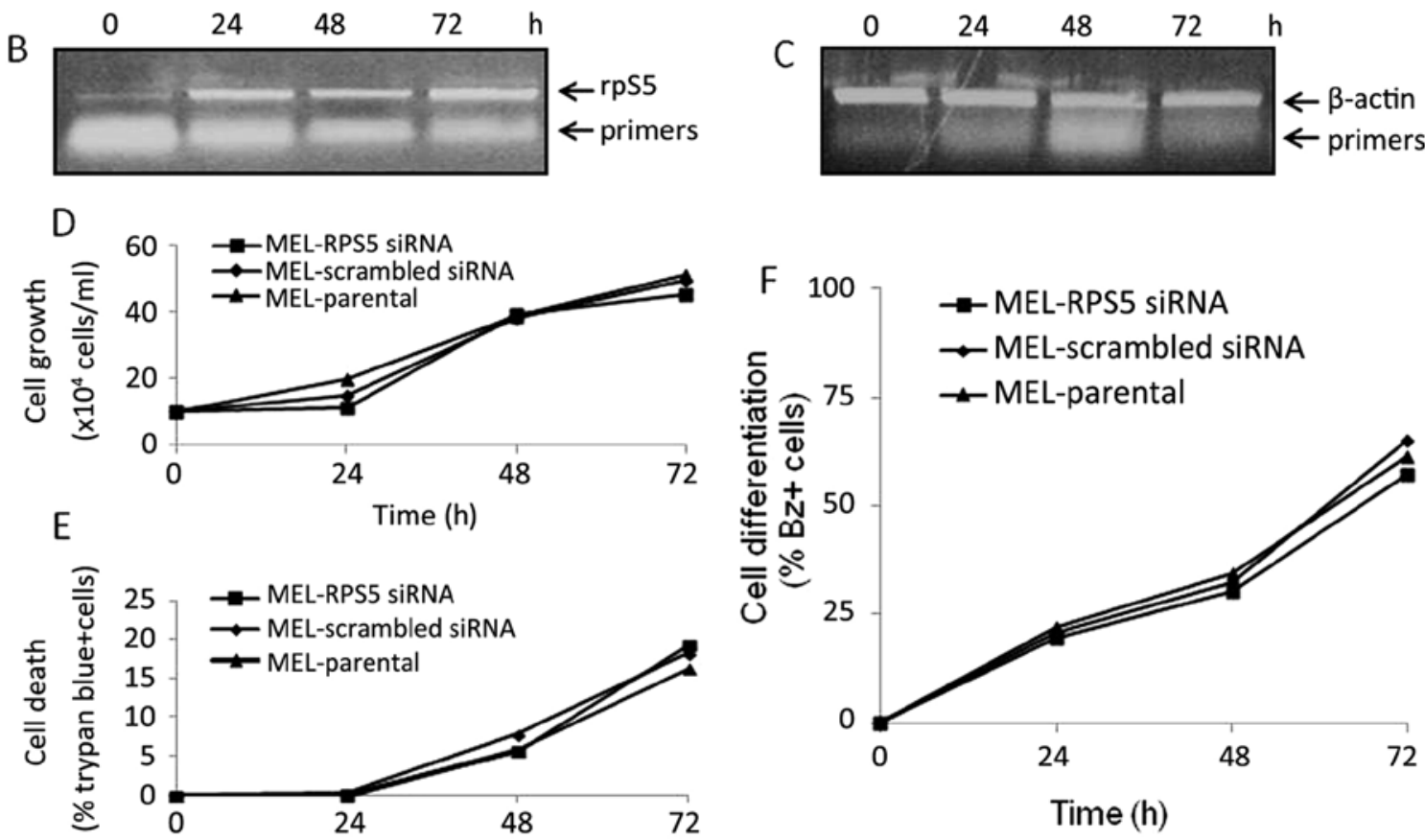

Figure 8. Assessment of differentiation potential of parental MEL cultures restoring normal RPS5 gene expression levels after an initial RNAi-mediated silencing of endogenous RPS5. Parental MEL cells were treated with either scrambled (MEL-scrambled siRNA) or RPS5 specific (MEL-RPS5 siRNA) siRNAs as indicated in Fig. 6 for $48 \mathrm{~h}$. At that time and before the addition of inducer HMBA $(5 \mathrm{mM})(0 \mathrm{~h})$, the medium was replaced with siRNAs-free fresh one. Note that parental MEL cells treated without siRNA molecules (MEL parental) and exposed to HMBA were also included in this experimental design as control. The kinetics of cell growth (D), differentiation (F) and dead (E) cells was assessed as shown in Fig. 6 and described in Materials and methods. Simultaneously and at time intervals shown (B and C), isolation of total cytoplasmic RNA was carried out to assess the expression of RPS5 (B) by RT-PCR. The assessment of $\beta$-actin mRNA (C) was also performed and used as control. Note that RPS5 gene expression levels were low (0 h) upon addition of HMBA and then recovered afterwards to much higher levels. The data shown are from a representative experiment repeated at least twice and the data represent the mean of two independent measurements.

phase arrest. In order to assess that propensity of MEL cells in both $R P S 5$-specific siRNAs- and/or scrambled siRNAstreated cultures, the accumulation of cells to $G_{0} / G_{1}$ was evaluated upon differentiation in vitro. No substantial difference between the two cultures was recorded in the 24-72-h period tested (data not shown). However, a difference in the differentiation potential in the two cell cultures was previously seen and discussed regarding the inability to commit only in cells treated with RPS5-specific siRNAs. We reasoned additionally to assess the accumulation of apoptotic cells upon induction of differentiation with HMBA. Indeed, as shown in Fig. 7, by using flow cytometry to assess Annexin V-positive cells, we were able to evaluate the kinetics of apoptotic cells accumulated in these cultures for the entire 24-96-h period. MEL-RPS5siRNA culture from the beginning of the induction of differentiation showed much lower proportion of cells exhibiting a pre-apoptotic or apoptotic phenotype. Interestingly, MEL cells treated with scrambled siRNAs accumulate $\sim 32 \%$ of both pre-apoptotic and apoptotic cells after $96 \mathrm{~h}$, whereas specific RPS5 siRNAs-treated cells only $\sim 17 \%$ during the same period.

The fact that the modulated gene expression level of RPS5 is associated with an altered differentiation capacity of MEL cell cultures in vitro (MEL-C14, MEL-antisenseRPS5 and MEL-RPS5 siRNA treated cells) has clearly proposed a correlation between MEL cell differentiation potential and RPS5 gene expression. In order to more precisely intercorrelate such connection, an attempt was made to restore the normal RPS5 expression levels in MEL cultures that previously exhibited an RNAi-mediated RPS5 gene silencing and then reversely assess their capacity to commit to erythroid maturation. In doing so, the experiment shown in Fig. 8 was designed. MEL cells were exposed for $48 \mathrm{~h}$ with either scrambled- or RPS5-specific siRNAs, before the replenishment of cultures with fresh medium containing only the inducer HMBA, no siRNAs (shown in Fig. 8A). MEL cells cultured in the presence of specific RPS5 siRNAs for $48 \mathrm{~h}$, were washed out and changed into fresh medium with the 
addition of inducer, restored their RPS5 gene expression levels within $24 \mathrm{~h}$ (Fig. 8B), whereas the level of $\beta$-actin mRNA used as control remained unchanged (Fig. 8C). However, such MEL cells exhibit their maximum differentiation potential comparable to that seen for both parental MEL and scrambled-siRNAs treated MEL cultures (Fig. 8F). Similarly, the kinetics of cellular proliferation in both terms of growth (Fig. 8D) and trypan-blue positive (dead) cells (Fig. 8E) are indistinguishable between the three differentiating MEL cultures. Overall, such data propose a close relationship between the recorded RPS5 gene expression level in MEL cells and their capacity to initiate the in vitro differentiation program to erythroid maturation.

\section{Discussion}

In this study and in order to more thoroughly investigate the function of RPS5 in the initiation of commitment to erythroid maturation and the onset of MEL differentiation program to accumulate haemoglobin-containing cells in culture, we stably transfected MEL cells with the full length mouse antisense RPS5 cDNA (MEL-antisenseRPS5) and also applied RNAi methodology to transiently silence the expression of RPS5 gene (MEL-RPS5SiRNA). The fact that overexpression of recombinant RPS5 in MEL-C14 cells abrogated their reprogramming to differentiation in vitro has shown a novel potential extraribosomal function of this protein in erythropoiesis (22). Indeed, this conclusion has been further supported through the establishment of MEL-antisenseRPS5 culture and the induction of differentiation upon exposure to chemical inducers. In particular, and comparatively to MEL-C14, these cells achieved lower onset of differentiation in culture, whereas they exhibited a delay in completing their erythroid maturation program in full (haemoglobin synthesis and limitation of proliferation potential). The latter effect would be attributed to the fact that although these cells do not produce full recombinant RPS5 RNA transcripts, they might accumulate, however, truncated antisense RPS5 molecules in the cytoplasm interfere with endogenous RPS5 mRNA to affect its function. A similar effect has been reported in the case of Notch, where the transfection of MEL cells with recombinant constructs of Notch antisense cDNA modulated the endogenous Notch mRNA level and function within the cells (30). The comprehensive analysis of the experimental data presented in this study indicated that although the cytoplasmic steady-state level of endogenous RPS5 RNA transcripts remains unchanged in differentiating MEL-antisenseRPS5 cells, the synthesis of the corresponding RPS5 protein dramatically decreases very early, soon after induction of differentiation as shown in this study, in contrast to MEL-C14 cells where this effect happened at latter stages of differentiation (22). Such a result implies that antisense-RPS5 RNA molecules may cause a translational inhibition of endogenous RPS5 mRNA, as seen in the case of antisense-Notch cDNA transfection in MEL cells, leading to modulated RPS5 function in the timed reprogramming of these cells to differentiate. More importantly, the causal relationship between the RPS5 gene expression and the differentiation potential of MEL cells is undoubtedly supported by the experiments presented in this study based on the selective RNAi-mediated silencing of RPS5 gene. By exhibiting silencing of RPS5 gene, the inability of such MEL culture to initiate commitment to erythroid maturation in vitro has been strikingly reversed by allowing cells to restore normal RPS5 gene expression level. The latter, confirms previous observations showing that imbalance in differentiation potential and differentiation-dependent apoptosis is correlated with the capacity of cells to continue proliferation in culture $(13,14)$. On the other hand, the cellular morphology showing a distortion of nucleus and nucleolus, coincides with an imbalance between the need for either proliferation (neoplasm phenotype), or differentiation/apoptosis decisions (erythroid maturation phenotype). Such a conclusion is in accordance with previously published data showing that MEL cell differentiation in vitro is associated very early with a noticeable decrease in the expression of RPS5 gene, as well as a sharp inactivation of the transcription of rRNAs genes $(15,16)$. Being also aware on the highly coordinated nature of the MEL erythroid maturation program regarding the transcriptional activation and/or repression of crucial genes, it is reasonable to assume that somehow the deregulation of RPS5 contributes to an imbalance in ribosomal biogenesis and function. It has been reported that the blockade of MEL erythroid differentiation program by methylation inhibitors (neplanocin A, 3-deaza-neplanocin A, and cycloleucine), caused constitutive expression of RPS5, thus implying a differentiation-dependent regulation for RPS5 gene in these cells (31).

The ribosomal proteins are thought to have mainly structural role to facilitate the proper configuration of rRNAs as integral moieties of ribosomal subunits, thereby promoting the speed and accuracy of the translation process (32). This conservative concept, however, is changing, by knowing that besides their profound structural and functional role in ribosome integrity and protein synthesis, ribosomal proteins exert extraribosomal roles in gene transcription, cell proliferation, apoptosis and differentiation (33). Also recently, an intense interest in delineating the role of ribosome function in the pathogenesis of certain blood disorders has been emerged. Indeed, ribosomal proteins have unexpectedly been found to contribute in a series of pathological conditions such as Diamond-Blackfan anemia (DBA) or 5q- syndrome. The latter happened at a rate that led to the term 'ribosomopathies' which has been coined to describe these and other syndromes characterized by abrogated ribosome biogenesis (21). Interestingly, the molecular pathogenesis of DBA depends on mutations in a number of different ribosomal protein genes with that of RPS19 accounting for $25 \%$ of DBA patients (34). The striking specificity of the defect manifestation mainly on red blood cell precursors is still elusive, while the list of ribosomal protein genes found mutated is growing to include members of the large as well the small ribosomal subunit such as RPS24, RPS17, RPS10, RPS26, RPL35a, RPL5 and $R P L 11$ (35-39). On the other hand, RPS14 is directly linked to $5 \mathrm{q}^{-}$syndrome pathogenesis, which presents many similarities to the clinical symptoms of DBA (40). Haploinsufficiency of certain ribosomal protein genes in Drosophila has been associated with a growth restricted phenotype called 'minute', while in Zebrafish it leads to tumorigenesis by an unknown mechanism (41). One of the major events during erythroid differentiation is the modulation of the number of ribosomes thus ensuring the synthesis of large amounts of haemoglobin 
needed for red blood cell function (13). The pathophysiology at the molecular level correlating to the selective dysfunction of the erythroid cell lineage with disorders of ribosome biogenesis is still elusive besides the fact that the p53 activation and p21 accumulation has been shown to cause cell cycle arrest in such ribosome abnormal erythroid progenitor cells. The latter, proposes that the erythroid lineage has a low threshold for the induction of $\mathrm{p} 53$, thus providing a basis for the failure of erythropoiesis in disorders like $5 \mathrm{q}^{-}$syndrome and DBA (42). A clear connection between erythropoiesis, ribosome biogenesis and specific ribosomal protein function is still elusive. We do not know precisely how ribosomal proteins contribute to the formation of the $40 \mathrm{~S}$ and $60 \mathrm{~S}$ ribosomal subunits and to which extent they contribute to the protein synthesis. In MEL cells for example, it has been proposed that ribosomal proteins are added sequentially during the formation of the small (40S) ribosomal subunit (43). In particular, RPS5 together with RPS4 and RPS12 have been implicated in the signaling step for the formation of a peptide bond (after the binding of tRNA in the ribosome) during the translation process (44). For example, the targeted disruption of mouse PRS19 gene that was lethal prior to implantation, thus exhibiting a potential role in erythropoiesis and development (45). Moreover, it is also noteworthy that RPS3 can act as a potential receptor for chemical inducers that initiate MEL cell differentiation in vitro, thus enriching our knowledge regarding an important extraribosomal functional role of this specific protein within cancer cells (46).

Initiation of MEL cell differentiation has been reported to occur within the $G_{1} / S$ interphase and that terminal erythroid cell maturation is associated with $\mathrm{G}_{0} / \mathrm{G}_{1}$ cell cycle arrest and the coordinated function of CDK2, CDK4 and CDK6 along with their inhibitors (CDKIs) $(13,14,25-28)$. As reported earlier, MEL-C14 cells delayed the initiation of the onset of differentiation upon exposure to chemical inducers, whereas at the same time a slower entrance of cells in $\mathrm{G}_{0} / \mathrm{G}_{1}$ phase and significant changes in the profile of CDK2, CDK4 and CDK6 have been recorded (22). Such a conclusion also applies for differentiating MEL-antisenseRPS5 cells as reported in this study, where a delay in the onset of initiation of differentiation has been uncovered with simultaneous significant alterations in the protein levels of individual CDKs. Alternatively, the deregulation in cell cycle and differentiation seen upon modulating RPS5 gene expression in MEL cells could be attributed to the altered c-myc expression profile seen in this study. Indeed, recent studies have clearly indicated the existence of a molecular link connecting cell growth control and ribosome biogenesis, as well as ribosome dysfunction and cell cycle regulation $(47,48)$. In particular, $c$ - myc plays a major role in balancing ribosome component production, whereas impairment of ribosome biogenesis (e.g., deregulation of RPL11 and RPL5) leads to p53 induction thus hindering cell cycle progression. Whether such a connection already exists for RPS5 in MEL cells is still elusive. As shown in this study, the extra-ribosomal function of RPS5 related to the modulation of the onset of MEL cell differentiation program must be attributed to the latent period before the initiation of commitment. This is an interesting, and intriguing possibility requiring further experimental verification and validation to provide insights into how RPS5 exerts its roles for crucial cellular decisions, such as proliferation, differentiation and apoptosis. In a previous study, the selective repression of RPS19 gene expression in human $\mathrm{CD} 4^{+}$cells caused abnormal ribosomal biogenesis that consequently has been clearly correlated with irregular cell cycle arrest and lineage-specific defects of erythroid progenitors (49). The data presented in this study contribute to the better understanding of the role of RPS5 in the initiation of commitment of MEL cells to terminal erythroid maturation. Moreover, such results provide new knowledge on the borderlines of erythropoiesis on how the pathogenesis of ribosomopathies and the modulated gene expression of RPS5 could impair red blood cell production and the function of haemoglobin. This is considered very attractive especially in light of evidence (50) showing that individual components of the translation machinery are deregulated in cancer cells, an event that also suggests the therapeutic exploitation of specific ribosome-related molecules as candidate targets for cancer therapy.

\section{Acknowledgements}

We would like to thank Elsa P. Amanatiadou (Ph.D. Fellow, Laboratory of Pharmacology, Department of Pharmaceutical Sciences at Aristotle University of Thessaloniki) for her substantial help both in experimentation and upon the preparation of this manuscript. This study was supported by interdepartmental funds and the Research Committee of the Aristotle University of Thessaloniki (no. 88074 to I.S.V.).

\section{References}

1. Adams GB and Scadden DT: The hematopoietic stem cell in its place. Nat Immunol 7: 333-337, 2006.

2. Moore KA and Lemischka IR: Stem cells and their niches. Science 311: 1880-1885, 2006.

3. Stamatoyannopoulos G: Control of globin gene expression during development and erythroid differentiation. Exp Hematol 33: 259-271, 2005.

4. Orkin SH and Zon LI: Hematopoiesis: An evolving paradigm for stem cell biology. Cell 132: 631-644, 2008.

5. Palis J: Ontogeny of erythropoiesis. Curr Opin Hematol 15: $155-161,2008$.

6. Tsiftsoglou AS, Vizirianakis IS and Strouboulis J: Erythropoiesis Model systems, molecular regulators, and developmental programs. IUBMB Life 61: 800-830, 2009.

7. Tsiftsoglou AS, Bonovolias ID and Tsiftsoglou SA: Multilevel targeting of hematopoietic stem cell self-renewal, differentiation and apoptosis for leukemia therapy. Pharmacol Ther 122: 264-280, 2009.

8. Edling CE and Hallberg B: c-Kit - a hematopoietic cell essential receptor tyrosine kinase. Int J Biochem Cell Biol 39: 1995-1998, 2007.

9. Elliott S, Pham E and Macdougall IC: Erythropoietins: A common mechanism of action. Exp Hematol 36: 1573-1584, 2008.

10. Zhang CC and Lodish HF: Cytokines regulating hematopoietic stem cell function. Curr Opin Hematol 15: 307-311, 2008.

11. Rice KN and Jamieson CH: Molecular pathways to CML stem cells. Int J Hematol 91: 748-752, 2010.

12. List AF, Vardiman J, Issa JP and DeWitte TM: Myelodysplastic syndromes. Hematology (Am Soc Hematol Educ Program) 2004: 297-317, 2004.

13. Tsiftsoglou AS, Pappas IS and Vizirianakis IS: Mechanisms involved in the induced differentiation of leukemia cells. Pharmacol Ther 100: 257-290, 2003

14. Tsiftsoglou AS, Pappas IS and Vizirianakis IS: The developmental program of murine erythroleukemia cells. Oncol Res 13: 339-346, 2003.

15. Tsiftsoglou AS, Wong W, Volloch V, Gusella J and Housman D: Commitment of murine erythroleukemia (MEL) cells to terminal differentiation is associated with coordinated expression of globin and ribosomal genes. Prog Clin Biol Res 102A: 69-79, 1982. 
16. Vizirianakis IS, Pappas IS, Gougoumas D and Tsiftsoglou AS: Expression of ribosomal protein S5 cloned gene during differentiation and apoptosis in murine erythroleukemia (MEL) cells. Oncol Res 11: 409-419, 1999.

17. Pappas IS, Vizirianakis IS and Tsiftsoglou AS: Cloning, sequencing and expression of a cDNA encoding the mouse L35a ribosomal protein during differentiation of murine erythroleukemia (MEL) cells. Cell Biol Int 25: 629-634, 2001.

18. Housman D, Volloch V, Tsiftsoglou AS, Levenson R, Gusella JF, Kernen $J$ and Mitrani A: Analysis of the molecular basis of commitment in murine erythroleukemia (MEL) cells. In In Vivo and In Vitro Erythropoiesis: The Friend Cell System. Rossi GB (ed). Elsevier/North Holland Biomedical Press, Amsterdam, pp273-282, 1979.

19. Hensold JO, Barth-Baus D and Stratton CA: Inducers of erythroleukemic differentiation cause messenger RNAs that lack poly(A)-binding protein to accumulate in translationally inactive, salt-labile $80 \mathrm{~S}$ ribosomal complexes. J Biol Chem 271: 23246-23254, 1996.

20. Bessis M, Lessin LS and Beutler E: Morphology of the erythron. In: Hematology. Williams WJ, Beutler E, Erslev AJ and Lichtman MA (eds). McGraw-Hill, New York, NY, pp257-279, 1983.

21. Raiser DM, Narla A and Ebert BL: The emerging importance of ribosomal dysfunction in the pathogenesis of hematologic disorders. Leuk Lymphoma 55: 491-500, 2014.

22. Matragkou CN, Papachristou ET, Tezias SS, Tsiftsoglou AS, Choli-Papadopoulou T and Vizirianakis IS: The potential role of ribosomal protein S5 on cell cycle arrest and initiation of murine erythroleukemia cell differentiation. J Cell Biochem 104: 1477-1490, 2008

23. Vizirianakis IS, Wong W and Tsiftsoglou AS: Analysis of the inhibition of commitment of murine erythroleukemia (MEL) cells to terminal maturation by N6-methyladenosine. Biochem Pharmacol 44: 927-936, 1992.

24. Gougoumas DD, Vizirianakis IS, Triviai IN and Tsiftsoglou AS: Activation of Prn-p gene and stable transfection of Prn-p cDNA in leukemia MEL and neuroblastoma N2a cells increased production of $\operatorname{PrP}(\mathrm{C})$ but not prevented DNA fragmentation initiated by serum deprivation. J Cell Physiol 211: 551-559, 2007.

25. Hsieh FF, Barnett LA, Green WF, Freedman K, Matushansky I, Skoultchi AI and Kelley LL: Cell cycle exit during terminal erythroid differentiation is associated with accumulation of p2 $7^{\mathrm{Kip} 1}$ and inactivation of cdk2 kinase. Blood 96: 2746-2754, 2000.

26. Matushansky I, Radparvar F and Skoultchi AI: Manipulating the onset of cell cycle withdrawal in differentiated erythroid cells with cyclin-dependent kinases and inhibitors. Blood 96 2755-2764, 2000.

27. Matushansky I, Radparvar F and Skoultchi AI: Reprogramming leukemic cells to terminal differentiation by inhibiting specific cyclin-dependent kinases in G1. Proc Natl Acad Sci USA 97: 14317-14322, 2000.

28. Zhu L and Skoultchi AI: Coordinating cell proliferation and differentiation. Curr Opin Genet Dev 11: 91-97, 2001.

29. Vizirianakis IS, Pappas IS and Tsiftsoglou AS: Differentiationdependent repression of c-myc, B22, COX II and COX IV genes in murine erythroleukemia (MEL) cells. Biochem Pharmacol 63: 1009-1017, 2002.

30. Shelly LL, Fuchs C and Miele L: Notch-1 inhibits apoptosis in murine erythroleukemia cells and is necessary for differentiation induced by hybrid polar compounds. J Cell Biochem 73: 164-175, 1999.

31. Vizirianakis IS and Tsiftsoglou AS: Blockade of murine erythroleukemia cell differentiation by hypomethylating agents causes accumulation of discrete small poly(A)- RNAs hybridized to 3'-end flanking sequences of beta(major) globin gene. Biochim Biophys Acta 1743: 101-114, 2005.
32. Zimmermann RA: The double life of ribosomal proteins. Cell 115: 130-132, 2003.

33. Wool IG: Extraribosomal functions of ribosomal proteins. Trends Biochem Sci 21: 164-165, 1996.

34. Draptchinskaia N, Gustavsson P, Andersson B, Pettersson M, Willig TN, Dianzani I, Ball S, Tchernia G, Klar J, Matsson H, et al: The gene encoding ribosomal protein S19 is mutated in Diamond-Blackfan anaemia. Nat Genet 21: 169-175, 1999.

35. Cmejla R, Cmejlova J, Handrkova H, Petrak J and Pospisilova D: Ribosomal protein S17 gene (RPS17) is mutated in DiamondBlackfan anemia. Hum Mutat 28: 1178-1182, 2007.

36. Gazda HT, Grabowska A, Merida-Long LB, Latawiec E, Schneider HE, Lipton JM, Vlachos A, Atsidaftos E, Ball SE, Orfali KA, et al: Ribosomal protein S24 gene is mutated in Diamond-Blackfan anemia. Am J Hum Genet 79: 1110-1118, 2006.

37. Farrar JE, Nater M, Caywood E, McDevitt MA, Kowalski J, Takemoto CM, Talbot CC Jr, Meltzer P, Esposito D, Beggs AH, et al: Abnormalities of the large ribosomal subunit protein, Rpl35a, in Diamond-Blackfan anemia. Blood 112: 1582-1592, 2008.

38. Gazda HT, Sheen MR, Vlachos A, Choesmel V, O'Donohue MF, Schneider H, Darras N, Hasman C, Sieff CA, Newburger PE, et al: Ribosomal protein L5 and L11 mutations are associated with cleft palate and abnormal thumbs in Diamond-Blackfan anemia patients. Am J Hum Genet 83: 769-780, 2008.

39. Doherty L, Sheen MR, Vlachos A, Choesmel V, O'Donohue MF, Clinton C, Schneider HE, Sieff CA, Newburger PE, Ball SE, et al: Ribosomal protein genes RPS10 and RPS26 are commonly mutated in Diamond-Blackfan anemia. Am J Hum Genet 86: 222-228, 2010.

40. Ebert BL, Pretz J, Bosco J, Chang CY, Tamayo P, Galili N, Raza A, Root DE, Attar E, Ellis SR, et al: Identification of RPS14 as a 5qsyndrome gene by RNA interference screen. Nature 451: 335-339, 2008.

41. Perry RP: Balanced production of ribosomal proteins. Gene 401: $1-3,2007$.

42. Dutt S, Narla A,Lin K, Mullally A, Abayasekara N, Megerdichian C, Wilson FH, Currie T, Khanna-Gupta A, Berliner N, et al: Haploinsufficiency for ribosomal protein genes causes selective activation of p53 in human erythroid progenitor cells. Blood 117: 2567-2576, 2011.

43. Todorov IT, Noll F and Hadjiolov AA: The sequential addition of ribosomal proteins during the formation of the small ribosomal subunit in Friend erythroleukemia cells. Eur J Biochem 131: 271-275, 1983.

44. Purohit $P$ and Stern S: Interactions of a small RNA with antibiotic and RNA ligands of the 30S subunit. Nature 370: 659-662, 1994.

45. Matsson H, Davey EJ, Draptchinskaia N, Hamaguchi I, Ooka A, Levéen P, Forsberg E, Karlsson S and Dahl N: Targeted disruption of the ribosomal protein S19 gene is lethal prior to implantation. Mol Cell Biol 24: 4032-4037, 2004.

46. Webb Y, Zhou X, Ngo L, Cornish V, Stahl J, ErdjumentBromage H, Tempst P, Rifkind RA, Marks PA, Breslow R, et al: Photoaffinity labeling and mass spectrometry identify ribosomal protein S3 as a potential target for hybrid polar cytodifferentiation agents. J Biol Chem 274: 14280-14287, 1999.

47. Lempiäinen $\mathrm{H}$ and Shore D: Growth control and ribosome biogenesis. Curr Opin Cell Biol 21: 855-863, 2009.

48. Teng T, Thomas G and Mercer CA: Growth control and ribosomopathies. Curr Opin Genet Dev 23: 63-71, 2013.

49. Kuramitsu M, Hamaguchi I, Takuo M, Masumi A, Momose H, Takizawa K, Mochizuki M, Naito S and Yamaguchi K: Deficient RPS19 protein production induces cell cycle arrest in erythroid progenitor cells. Br J Haematol 140: 348-359, 2008.

50. Ruggero D and Pandolfi PP: Does the ribosome translate cancer? Nat Rev Cancer 3: 179-192, 2003. 